\section{A universal food-paste dispenser for use with cats and other animals}

\section{MARK A. BERKLEY, F. T. CRAWFORD, and GRAHAM OLIFF \\ Florida State University, Tallahassee, Florida 32306}

A description is provided for a relatively inexpensive universal food-paste dispenser which can be used with cats and other animals.

When attempting to train animals to emit responses for a reward, the choice of the reward to be used is often determined by the devices available to dispense it. Among such devices, the pellet dispenser is probably the most popular and convenient. Pellet dispensers, however, require specially prepared rewards which are: (1) not adequate reinforcers for many animals, (2) moderately expensive, (3) too difficult to make, and (\$) available only in a limited number of varieties (flavors). One flavor notably lacking in available pellets is meat. Carnivores, many birds, and reptiles will work well for meat rewards, but meat flavored pellets are very difficult to make (Berkley, 1970). Fresh meat tidbits are tedious to make, spoil easily, and are difficult to dispense.

Meat and fish flavored slurries have been described (e.g., Hodos, Laursen, \& Nissen, 1963; Crawford \& Kenshalo, 1965) and may be dispensed with a conventional liquid dipper feeder. Preparing and keeping slurries is an onerous task, as is the cleaning and maintaining of the dispenser. Syringe feeders such as the Davis pump or Hulse feeder (Hulse, 1960) are easily clogged by pastes and slurries because the food must pass through small orifices and relatively long tubing channels.

The device described in this note was specifically designed to permit the reliable delivery of meat pastes. It has a large reservoir and large orifices so that any food which can be reduced to a smooth paste (e.g., beef hearts, bananas, etc.) can be used as a reward. By selecting the most preferred food, the device has permitted the training of cats (Berkley, 1970), hawks, and vultures with surprising ease.

The feeder device (Fig. 1) consists of a solenoid-operated two-way air valve, a food piston and block, an air-operated piston, a food reservoir, and a compressed-air source. Compressed air is passed through an air cleaner, lubricator, and pressure regulator (left side, Fig. 2), which reduces the pressure to about $40 \mathrm{psi}$. The air is led by pressure tubing to a solenoid valve (E, Fig. 2). This electrically controlled valve has both a normally open and a normally closed port. When power is applied to the valve, the port states are reversed. Pressure tubing is connected from the exit ports of the valve to an air-operated piston (B, Figs. 1 and 2). The piston is kept in its extended position by air pressure from the normally open port of the solenoid valve. The air piston is coupled to another piston (A, Fig. 1), which moves in a hole in the feeder block (D, Fig. 2). In the rest position, this second piston is flush with the front surface of the feeder block. The feeder block has, in addition to the piston channel, a food reservoir channel ( $C$, Fig. 2). The outer end of this channel is threaded and receives the threaded exit tube of the food reservoir, as shown in C, Fig. 2. The food reservoir contains the food to be dispensed and is under slight pressure (1-3 psi) controlled by a second air regulator (F, Fig. 2). A free-floating Teflon piston inside the reservoir transmits the air pressure uniformiy to the food. The food reservoir, feeder block, and piston are constructed to be easily disassembled for cleaning.

To operate, an electrical pulse of about 100 msec duration is delivered

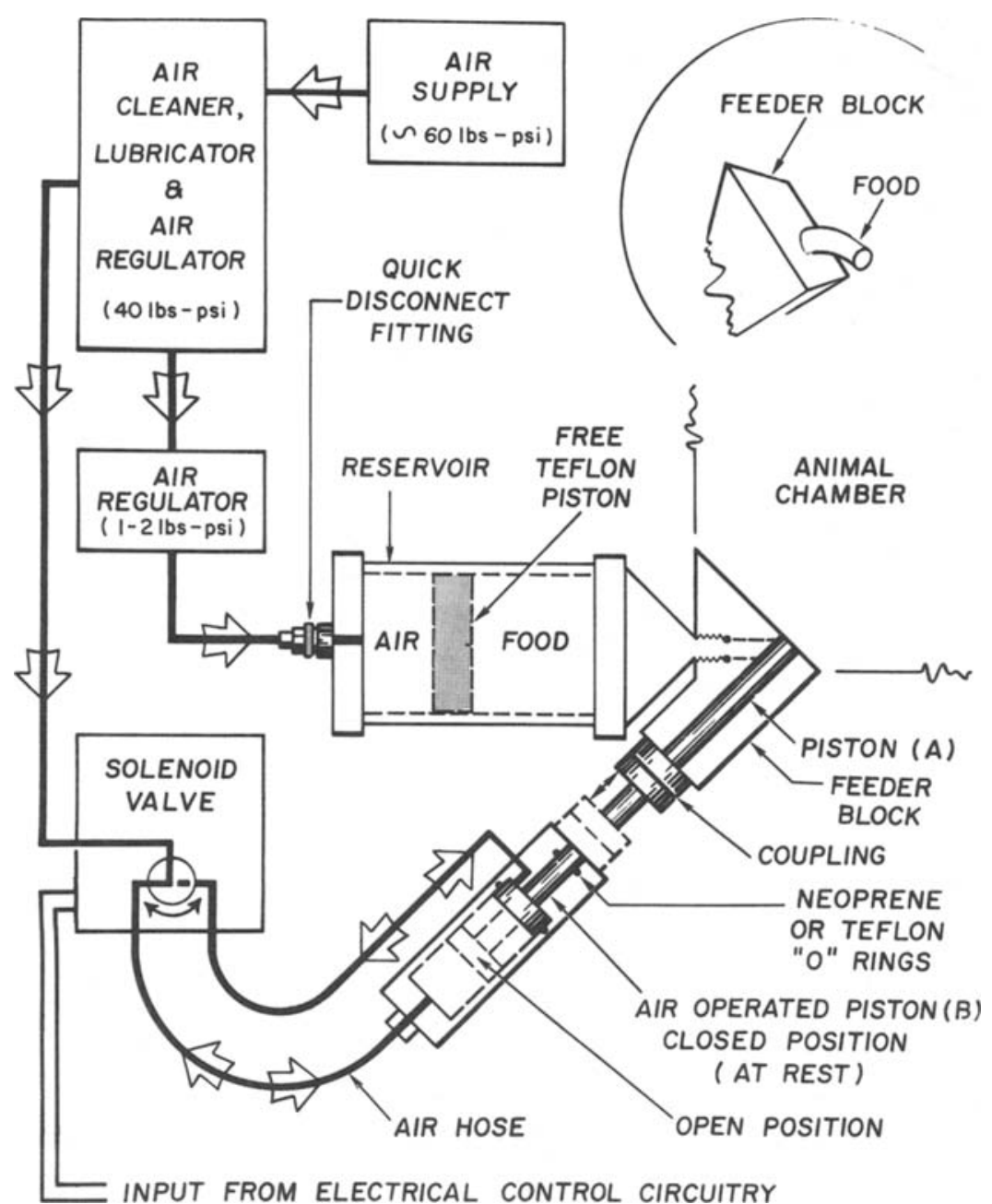

Fig. 1. General schematic of air-operated piston food-paste dispenser. Heavy black lines indicate air-pressure tubing and the arrows, the direction if air movement. The food reservoir, feeder block, and air-operated piston are mounted as a separate unit and may be located some distance from the air preparation and switching apparatus, as shown in Fig. 2. Upper right: Detail of front of feeder block showing extruded food. 


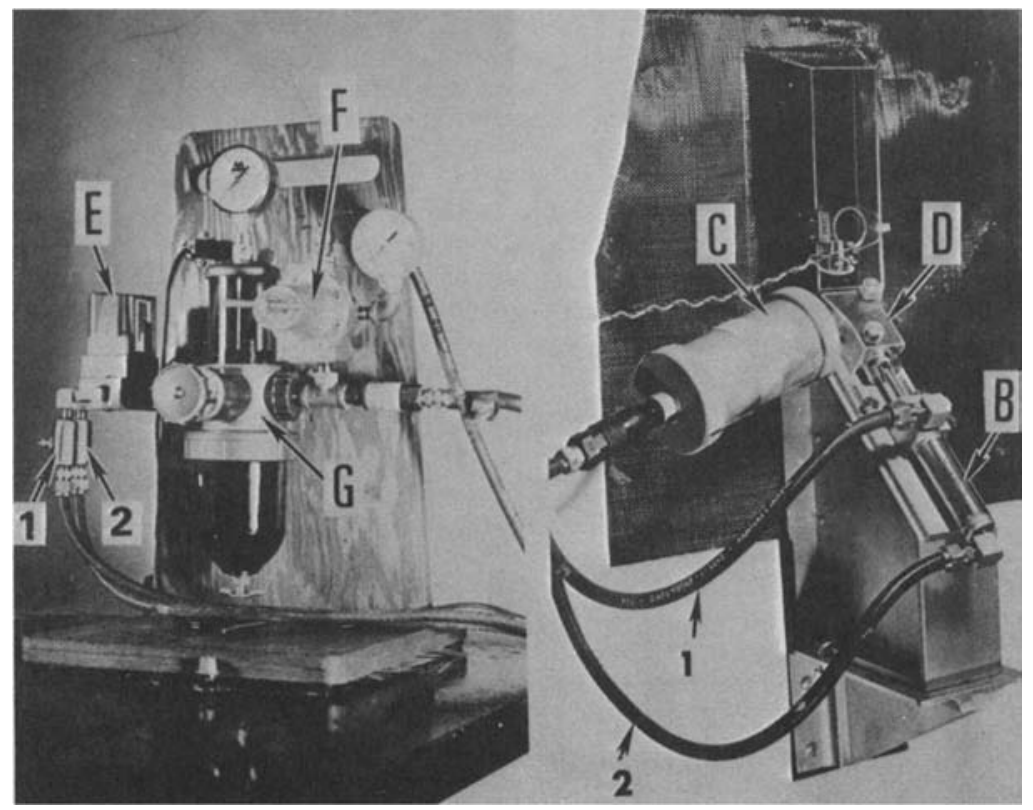

Fig. 2. Left side: Air preparation and switching apparatus. E-solenoid valve; $F$-food reservoir and air-pressure regulator and gauge; $G$-main air-pressure regulator with integral lubricator and water trap; 1 and 2 -needle valves. Right side: Rear view of food dispensing portion of apparatus, showing how parts are mounted. B-air-operated piston; C-food reservoir; $D$-feeder block with feeder piston rod.

Table 1

Parts List

Parker-Hannifin solenoid valve (GG 200-25)

$\$ 32.00$

Hannifin air piston-Universal Midget Series " $\mathrm{S}$ " style U.S. 1-in. stroke, $\% /$-in. bore

* Universal Air cleaner, regulator, and lubricator-Air Perfecting Service Co. B100A

Imperial-Eastman pressure tubing. c-403-1 rayon braid

Preu-trol needle valves $1 / 8$ in. N10B

Assorted brass fittings

Beckett-Harcum Co., Inc. air regulator 3R2-421

Aschroft (or Marsh Instrument Co.) low pressure gauge 0-15 lb

$\$ 12.00$

$\mathbf{\$ 3 7 . 5 0}$

$\$ .15 / \mathrm{ft}$

$\$ 3.00$

$\$ 10.00$

115.00

$\$ 3.00$

*C.W. Murphy, Inc., Perfecting Service Division, Charlotte, N.C. B100A

to the solenoid valve. This reverses the air-pressure relationship, releasing the pressure behind the air piston and increasing the pressure in front of it, forcing the air piston (B) and the coupled food piston (A) to move back momentarily. This opens the food channel from the food reservoir, and food oozes into the piston channel. When the electric pulse to the solenoid has ended, the pistons ( $A$ and $B$ ) return to their rest position and the food that oozed into the piston channel is ejected out of the food block where it can be consumed by the animal. (See detail at the upper right corner of Fig. 1). The amount of food ejected is determined by (1) the length of the electrical pulse, (2) the bore of the food piston channel,
(3) the rate at which the piston moves, (4) the consistency of the food paste, and (5) the air pressure in the food reservoir. It is most convenient to fix the value of all these variables except the food reservoir pressure. Thus, by changing food reservoir pressure, the reward size can be easily varied. Paste consistency is difficult to manipulate, so commercially available food pastes such as baby food are preferred because of the reliable consistency and the variety of foods available. The rate at which the piston moves may be controlled by placing needle valves to regulate airflow in the air lines going to the air piston (Fig. 2, left side, Nos. 1 and 2).

For the most reliable operation, Teflon " $O$ " rings should be used, and the reservoir, feeder block, and piston cleaned regularly. None of the dimensions appear to be critical, but we have found that a food piston diameter of $1 / 4$ in. and piston movement of about $3 / 4$ in. is best. The reservoir was made to hold about $4 \mathrm{oz}$ of food (two small bottles of baby food). All metal parts may be constructed of aluminum and anodized, but the feeder piston should be made of stainless steel so that it can withstand constant handling without damage. An idea of the scale and proportions can be obtained from Fig. 2. Several feeders may be operated from one regulator and lubricator, and this unit can be located some distance from the feeder, thus reducing the cost of the feeders considerably. Seven feeders have been constructed to date and have reliably operated several hundred thousand times without failure. A parts list is given in Table 1 , with a total cost of less than $\$ 125$. Plan details will be sent on request.

\section{REFERENCES}

BERKLEY, M. A. Visual discriminations in the cat. In $W$. Stebbins (Ed.). Animal psychophysics. New York Appleton-Century-Crofts, 1970.

CRAWFORD F T \&ENSHALO, D. $R$ A liquid reinforcer for the cat. Journal of the Experimental Analysis of Behavior, 1965, 8, 29-30.

HODOS, W. LAURSEN, A. M., \& NISSEN, T. A reinforcer for cats. Joumal of the Experimental Analy sis of Behavior, 1963, 6. 162 .

HULSE, S. H., JR. A precision liquid feeding system controlled by licking behavior. Journal of the Experimental Analysis of Behavior, 1960, 3, 1. 